Original Paper

\title{
Are there $E$ WS 1 ReARRANGED CUTANEOUS HIDRADENOMAS AND MUCOEPIDERMOID CARCINOMAS OF SALIVARY GLANDS? A FISH STUDY AND REVIEW OF THE LITERATURE
}

\author{
Onder Bozdogan ${ }^{1}$, Elçin Kadan ${ }^{1}$, Nazan Bozdogan ${ }^{2}$
}

${ }^{1}$ Department of Pathology, Gulhane Education and Research Hospital, Etlik, Ankara, Turkey

${ }^{2}$ Department of Pathology, Abdurrahman Yurtaslan Oncology Education and Research Hospital, Ankara, Turkey

\begin{abstract}
MAML2 rearrangements have been previously described for hidradenomas and mucoepidermoid carcinomas (MEC). However, one report showed EWSR1 rearrangement in both tumours. In this study, EWSR1 and MAML2 translocation were investigated in hidradenomas and MECs.

Specimens from thirteen MECs of the salivary glands and twenty hidradenomas of the skin were evaluated. Fluorescence in situ hybridisation (FISH) studies with EWSR1 and MAML2 break-apart probes were used.

Forty percent of hidradenomas and $84.6 \%$ of MECs showed a positive MAML2 break signal. EWSR1 break signal was absent in hidradenomas. Only two MECs showed positive EWSR1 signal and were, thus, reclassified as clear cell carcinoma (CCC). A statistically significant relationship was also observed between clear cells containing hidradenomas and the FISH status.

Despite the previous study, EWSR1 translocations could not be established in hidradenomas and MECs. The study further suggests that evaluation of EWSR1 might be obligatory for the correct diagnosis of $M A M L 2$-negative MECs to exclude the chance of CCCs. The present study also supports the notion that MAML2 can be used as a marker for hidradenomas and MECs.
\end{abstract}

Key words: MAML2, EWSR1, FISH, hidradenomas, mucoepidermoid carcinomas.

\section{Introduction}

Hidradenomas refer to benign tumours of the sweat glands. In general, hidradenomas are characterised by a complex morphology and the presence of a variety of cells. These tumours are composed of solid, cystic and glandular areas [1]. Besides these morphological patterns, hidradenomas also harbour $\mathrm{t}(11 ; 19)$ translocation, resulting in CRTC1-MAML2 fusion, which has been reported also in cases of mucoepidermoid carcinoma (MEC) of salivary glands [2].

In addition to CRTC1-MAML2 fusion, in 2008, Moller et al. reported the fusion of EWSR1 and POUSF1 genes in hidradenomas of the skin and
MECs of the salivary glands [3]. This rearrangement has also been described in a subgroup of soft tissue myoepitheliomas with distinctive clear cell morphology that occurs in children and young adults [4]. Despite Moller's study, the presence of EWSR1 rearrangement in hidradenomas and MECs is a controversial issue [3, 4, 5]. Furthermore, the presence of an EWSR1 translocation in clear cell carcinoma (CCC) of salivary glands, closely mimics MECs in routine practice, which increases its complexity [6].

This study aimed to clarify the presence of EWSR1 rearrangement in cutaneous hidradenomas and MECs of minor and major salivary glands with the fluorescence in situ hybridisation (FISH) technique and to 
evaluate the $M A M L 2$ status in these groups of tumours.

\section{Material and methods}

\section{Study group}

Specimens from thirteen MECs of the major and the minor salivary glands and twenty hidradenomas were included in this study. All hidradenoma cases

Table I. Study groups

\begin{tabular}{l} 
HidRADENOMA \\
\hline $\mathrm{n}=20$ \\
Gender: \\
$14 \mathrm{~F} / 6 \mathrm{M}$ \\
Age: \\
$\quad$ Mean \pm SD: $52.5 \pm 14.5$ \\
Range: $24-74$ year \\
Localisation: \\
$\quad$ Head and neck: 6 \\
Body: 6 \\
$\quad$ Upper extremities: 3 \\
Lower extremities: 5 \\
Diameter: \\
Mean \pm SD: $1 \pm 0.6$ \\
Range: $0.2-3.2 \mathrm{~cm}$
\end{tabular}

MuCOEPIDERMOID CARCINOMA
Gender:
$8 \mathrm{~F} / 5 \mathrm{M}$
Age:
$\quad$ Mean \pm SD: $47.2 \pm 20.8$
$\quad$ Range: $9-83$ years
Localisation:
$\quad$ Parotis: 10
Oral (Minor SG): 3
Diameter:
Mean \pm SD: $1.5 \pm 0.7$
Range: $0.7-3 \mathrm{~cm}$
Grade:
AFIP:
$\quad$ High: 1
Intermediate: 1
Low: 11

were re-evaluated and confirmed based on the new WHO classification of skin tumours. Poroid hidradenomas which are now classified under the poroma group were excluded from the study [7]. The morphological features of MECs were also evaluated and graded using the Armed Force Institute of Pathology grading (AFIP) scheme [8]. The clinical data for the tumour groups were obtained from hospital information systems. The details of the study groups are summarised in Table I.

\section{Ethics statement}

This study was financially supported by the Ankara Numune Research and Education Hospital Medical Speciality Education Board (TUEK, Project no. 217-0033). All the experiments were conducted according to the institutional ethical guidelines (Ankara Numune Research and Education hospital Local Ethics Committee, 26.04.2017, 1383/2017).

\section{Fluorescence in situ hybridisation (FISH) study}

FISH studies were performed by using a ZytoLight SPEC EWSR1 Dual Colour Break Apart Probe (Zytovision, Bremerhaven, Germany) and a ZytoLight SPEC MAML2 Dual Colour Break Apart Probe. All analyses and evaluations were carried out using a Nikon Eclipse 80i fluorescence microscope (Nikon Europe, Amsterdam, Netherlands). A total of 100 non-overlapping intact nuclei were scored using Nikon NIS Elements 3.0 software (Nikon Europe, Amsterdam, Netherlands), and a cut-off value of $>10 \%$ of neoplastic cells showing the break-apart signal was used for analysis.

\section{Statistics}

The $\chi^{2}$ test using the PASW 17 statistic programme (Chicago, USA) was used to analyse the relationship between morphological details of the hidradenomas and FISH status. Because the minimum expected count was less than 5 for all comparisons, Fisher's Exact Test was used. The comparison between FISH-positive and FISH-negative hidradenomas for age, diameter and mitotic count was established with the help of the Mann-Whitney U test.

\section{Results}

The FISH analysis of hidradenoma specimens revealed the presence of $M A M L 2$ break-apart signal in eight hidradenomas (40\%), but the EWSR1 breakapart signal was completely absent.

For $M A M L 2$, the expected signal pattern of one orange-green fusion $(\mathrm{F})$, one orange $(\mathrm{O})$ and one separate green signal $(\mathrm{G})(1 \mathrm{~F} 1 \mathrm{O} 1 \mathrm{G})$ was observed in $14-83 \%$ of the cells of hidradenomas. Furthermore, unusual expression patterns were also observed which could be attributed to polysomy of the tumour cells. 
Table II. Morphological and clinical features of FISH(+) and FISH(-) hidradenomas*

\begin{tabular}{|c|c|c|c|}
\hline & FISH $(+)$ & FISH (-) & TOTAL \\
\hline Gender & $5 \mathrm{~F} / 3 \mathrm{M}$ & $9 \mathrm{~F} / 3 \mathrm{M}$ & $14 \mathrm{~F} / 6 \mathrm{M}$ \\
\hline \multirow[t]{2}{*}{ Age (mean $\pm \mathrm{SD})$} & $43.7 \pm 12.8$ years & $58.4 \pm 12.9$ years & $52.5 \pm 14.5$ years \\
\hline & $24-65$ years & $35-74$ years & $24-74$ years \\
\hline \multirow[t]{4}{*}{ Localisation } & Head and neck: 2 & Head and neck: 4 & Head and neck: 6 \\
\hline & Body: 4 & Body: 2 & Body: 6 \\
\hline & Upper ext.: 1 & Upper ext.: 2 & Upper ext.: 3 \\
\hline & Lower ext.: 1 & Lower ext.: 4 & Lower ext.: 5 \\
\hline \multirow[t]{2}{*}{ Diameter $($ mean $\pm \mathrm{SD})$} & $1 \pm 0.4 \mathrm{~cm}$ & $0.9 \pm 0.7 \mathrm{~cm}$ & $1 \pm 0.6 \mathrm{~cm}$ \\
\hline & $0.5-2 \mathrm{~cm}$ & $0.2-3.2 \mathrm{~cm}$ & $0.2-3.2 \mathrm{~cm}$ \\
\hline \multirow[t]{3}{*}{ Dermal (D)/subcutaneous (SC) } & D: 4 & D: 8 & $\mathrm{D}: 12$ \\
\hline & SC: 0 & SC: 0 & SC: 0 \\
\hline & D/SC: 4 & D/SC: 4 & D/SC: 8 \\
\hline Tubule & $5 / 8$ & $5 / 12$ & $10 / 20$ \\
\hline Cysts & $6 / 8$ & $10 / 12$ & $16 / 20$ \\
\hline Polygonal cells & $8 / 8$ & $12 / 12$ & $20 / 20$ \\
\hline Clear cells & $8 / 8$ & $3 / 12$ & $11 / 20$ \\
\hline Squamoid cells & $1 / 8$ & $5 / 12$ & $6 / 20$ \\
\hline Sebaceous cells & $0 / 8$ & $1 / 12$ & $1 / 20$ \\
\hline Mucinous cells & $0 / 8$ & $1 / 12$ & $1 / 20$ \\
\hline Poroid areas/cells & $0 / 8$ & $4 / 12$ & $4 / 20$ \\
\hline Calcification & $0 / 8$ & $0 / 12$ & $0 / 20$ \\
\hline Decap. secret & $1 / 8$ & $2 / 12$ & $3 / 20$ \\
\hline Nuclear Groove & $6 / 8$ & $6 / 12$ & $12 / 20$ \\
\hline Necrosis & $0 / 8$ & $1 / 12$ & $1 / 20$ \\
\hline Epidermal connection** & $0 / 6$ & $1 / 9$ & $1 / 15$ \\
\hline \multirow[t]{2}{*}{ Stroma } & Hyalinized: 6 & Hyalinized: 9 & Hyalinized: 15 \\
\hline & Other: 2 & Other:3 & Other:5 \\
\hline Mitosis (mean $\pm \mathrm{SD}$ ) $10 \mathrm{HPF}$ & $0.8 \pm 1.1$ & $2.2 \pm 2.4$ & $1.7 \pm 2.1$ \\
\hline
\end{tabular}

* The morphological data for hidradenomas was evaluated on the basis of the morphologic features previously summarised by Nandeesh and Rajalakshmi [1]

** Epidermis cannot be detected in all of the cases due to surgical procedure

A statistically significant relationship was observed between clear cells containing hidradenomas and the FISH status ( $p=0.001)$. However, no significant relationship was observed between other observed morphological parameters, including cysts, tubule formation, nuclear grooves, decapitation secretions, dominant stroma type, mucinous, poroid and squamous cells (Table II). Clinically, FISH-positive hidradenomas were present in younger patients compared with FISH-negative ones (Mean: 43.7 vs. 58.4; $\mathrm{p}=0.031$ )

In the MEC group, 11 cases showed a MAML2 break signal (84.6\%), whereas the remaining two cases showed only an EWSR1 break signal. These 2 specimens were obtained from minor salivary glands.
As observed in the hidradenoma group, the expected signal pattern of $1 \mathrm{~F} 1 \mathrm{O} 1 \mathrm{G}$ for $M A M L 2$ was seen in $13-80 \%$ of the cells of MEC. Unusual expression patterns similar to hidradenomas were also detected. EWSR1 break signals of $72 \%$ and $83 \%$ of the cells were detected in the two FISH-positive MEC cases.

\section{Discussion}

In recent years, similar to salivary gland tumours, several molecular markers for skin adnexal tumours such as MAML2, have been identified with advances in molecular pathology $[9,10,11,12,13]$. This 

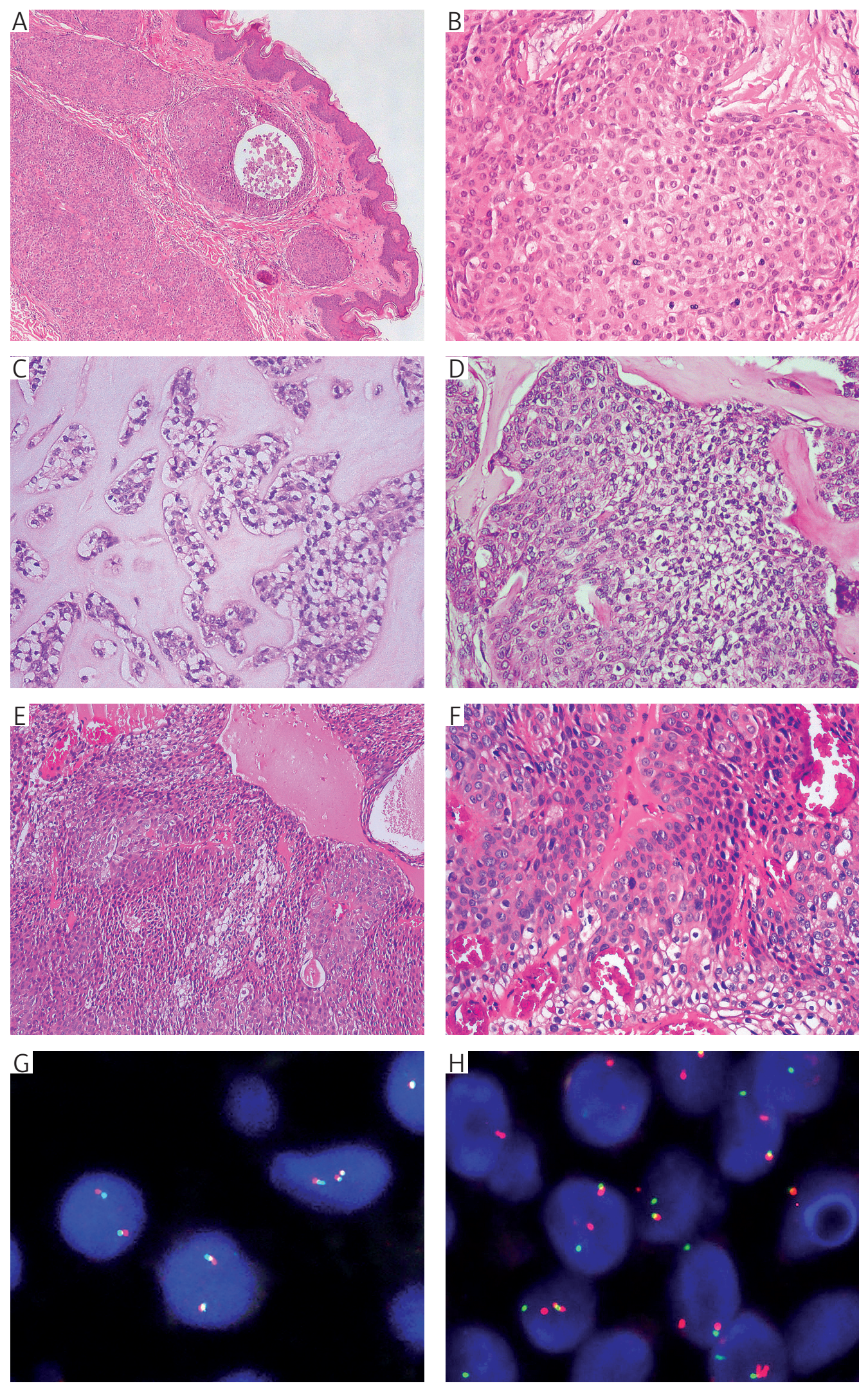

Fig. 1. Three hidradenoma cases positive for $M A M L 2$ FISH analysis. First case shows the presence of eosinophilic round cells, with focal areas indicative of hardly detectable clear cells (A; B). In the second case, both eosinophilic and clear cell islands can be seen embedded in the hyaline stroma (C; D). The third case includes cystic areas, and eosinophilic cell areas, and clear cells can also be easily detected (E, F). FISH studies of the first case clearly demonstrate a EWSR1-negative (G) but $M A M L 2$-positive (H) signal 

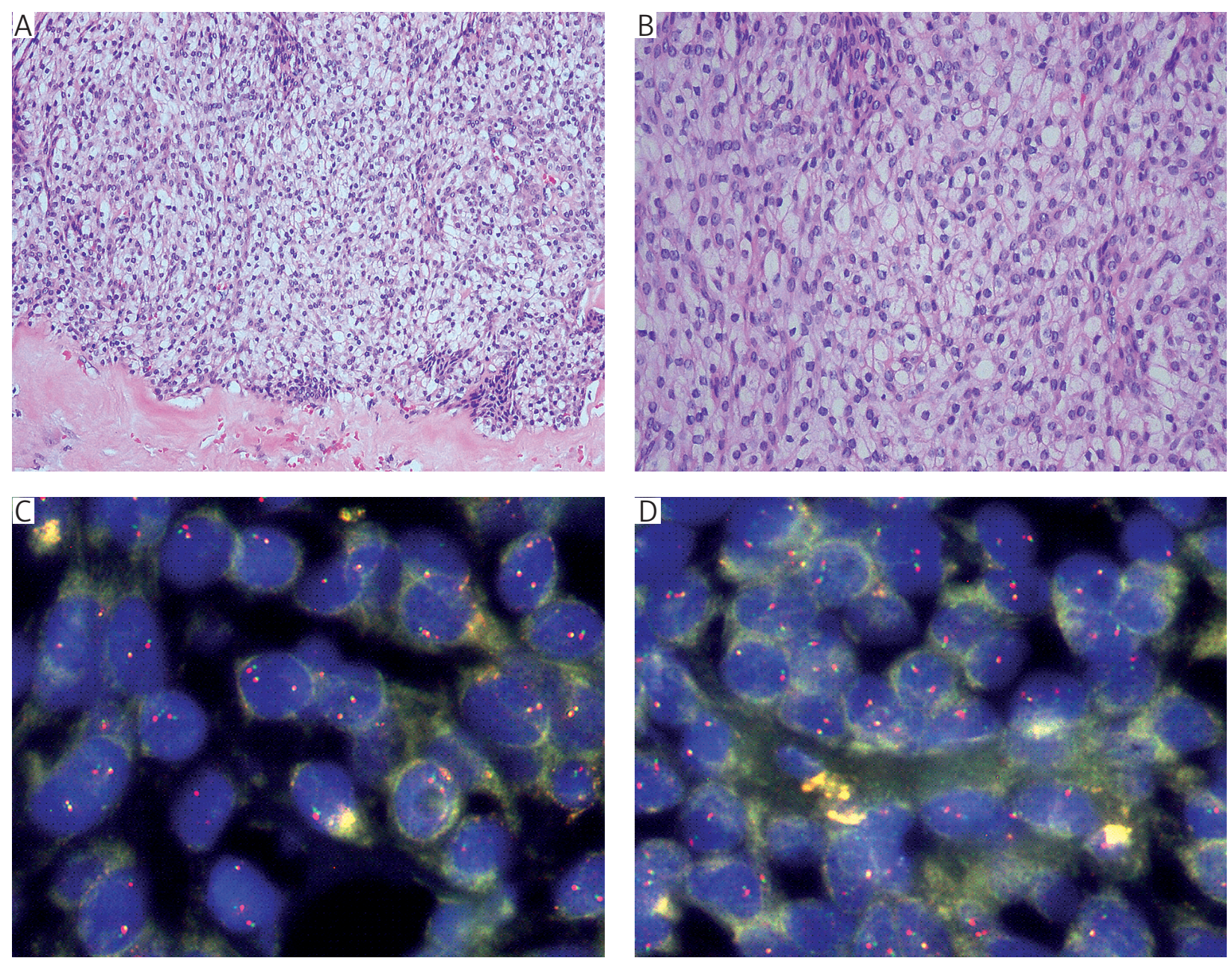

Fig. 2. Clear cell dominant hidradenoma (A; B) with negative EWSR1 (C) and MAML2 (D) FISH studies

indicates a close similarity between skin adnexal tumours and salivary gland neoplasms.

The $E W S R 1$ gene region is a common translocation partner in various soft tissue tumours [14]. However, its occurrence in epithelial tumours is rare. CCCs of salivary glands, clear cell odontogenic carcinomas and soft tissue myoepithelial carcinomas are well known examples of epithelial tumours with EWSR1 translocations $[15,16]$. EWSR1 translocation in MEC has remained a source of controversy in head and neck pathology. Moller et al. showed the presence EWSR1 rearrangements (EWSR1-POUSF1) in an 85-year-old man [3]. However, it is not clear whether these rearrangements were an EWSR1 translocation-positive MEC or not. Shah et al. and Antonescu et al. reported that none of the mucoepidermoid carcinomas in their studies demonstrated EWSR 1 rearrangements $[4,17]$. In a recent study involving $M A M L 2$ translocation negative MECs, 3 out of 17 MAML2 fusion-negative cases were reported to be positive for EWSR 1 translocation. However, these cases were reclassified as hyalinising CCC despite morphological similarity and MEC diagno- sis [5]. Similar to this study, our two cases originally diagnosed as MEC were EWSR1 translocation-positive. Both tumours were excised from the oral cavity. Immunohistochemically, both cases showed p63 positivity but no S100 expression. Cytokeratin 7 positivity was seen in one patient. These cases were morphologically re-evaluated by an experienced head and neck pathologist (see Acknowledgments). Therefore, these two cases were finally diagnosed as CCC (Fig. 3) with consensus. This is suggestive of a false diagnosis of CCC as MEC in the absence of FISH studies, particularly in cases of minor salivary glands.

The presence of EWSR1 rearrangement has been shown in cutaneous myoepithelial tumours including mixed tumour, myoepithelioma and myoepithelial carcinoma $[4,18]$. However, Moller et al. also reported EWSR1 translocation in 5 hidradenomas [3]. In our study, no EWSR1 rearrangement was detected in the hidradenomas of the skin. In another study, which focused on EWSR1 rearrangement in soft tissue myoepithelial tumours, Antonescu et al. reported that five cutaneous eccrine hidradenomas were used 

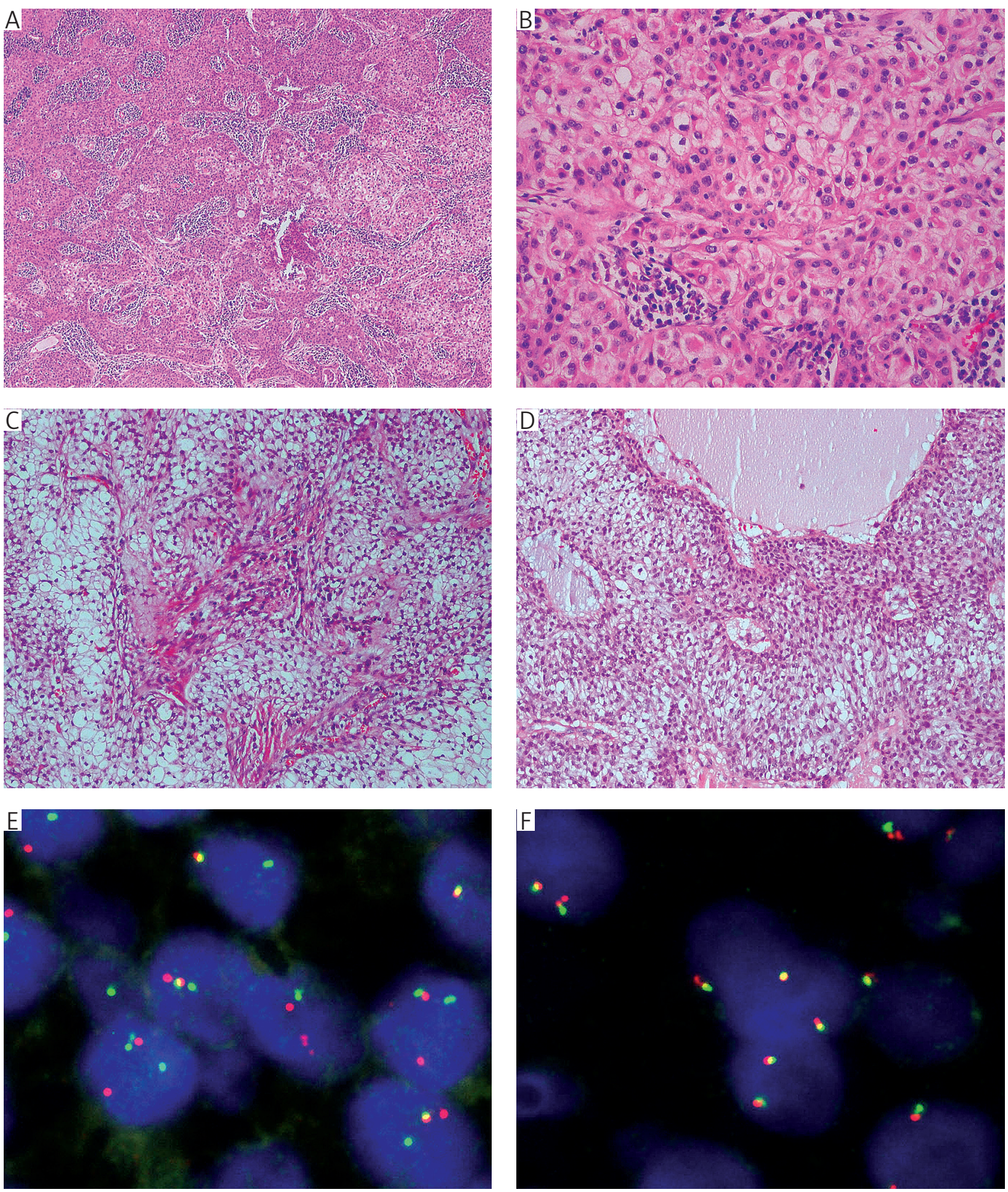

Fig. 3. Two salivary gland cancers positive for EWSRI fusion. The first case shows solid and trabecular areas of eosinophilic cells with focal clear cell areas (A; B). The second case contains clear cells with some cystic areas (C; D). EWSR1 FISH study of the second case clearly demonstrates a break signal in significant number of cells (E). However, no MAML2 break signal was detected (F)

as a control group and that none of them showed an abnormal EWSR1 gene. [4] Therefore, EWSR1 rearrangement is a very rare event in hidradenomas if it exists. Future studies involving larger groups might be helpful to explore this theory in detail.
Translocations involving MAML2 locus have been previously described for both MECs and hidradenomas $[19,20]$. The translocation of CRTC1-MAML2 has been found to be commonly associated with hidradenomas. Besides this, there are several reports on 

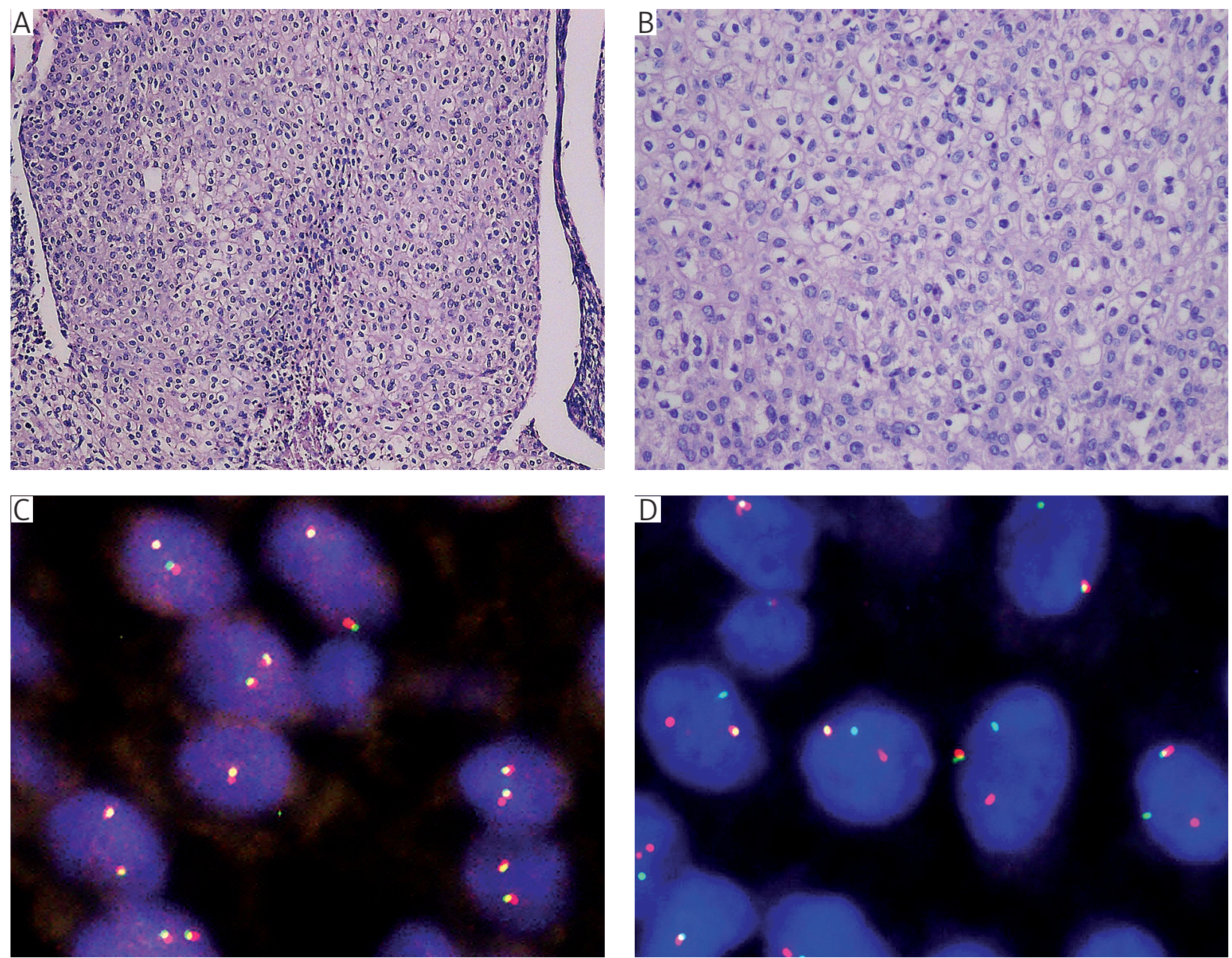

Fig. 4. Clear cell dominant MEC of parotid gland. Despite the presence of clear morphological similarities with EWSR1-positive cases, FISH studies show a EWSR1-negative (C) but MAML2-positive signal (D)

the occurrence of CRTC3-MAML2 fusion in a small group of tumours [2, 19]. In 1994, Gorunova et al. reported genetic alteration for this locus in hidradenomas for the first time. The study further pointed out $\mathrm{t}(11 ; 19)$ (q21;p13) translocation in one of the cases, in addition to other karyotypic changes [21]. In 2005, Behboudi et al. demonstrated $\mathrm{t}(11 ; 19)$ translocation in one hidradenoma case with the help of the RT-PCR technique [22]. Following this, several studies were conducted focussing on this issue $[2,12,19,23]$. In our study, $M A M L 2$ translocations were detected in $40 \%$ of the hidradenomas (Fig. 1). Similarly, several reports clearly highlight that approximately half of the hidradenomas are characterised by $M A M L 2$ translocations $[2,12,19,23]$.

It is important questions to evaluate whether any morphological and/or clinical differences exist between fusion-positive and fusion-negative hidradenomas. In this study, a statistically significant correlation was observed between clear cells of hidradenoma and $M A M L 2$ translocation status $(\mathrm{p}=0.001)$. Similar results were reported by Winnes et al., whose studies showed the presence of more or less abundant areas of clear cells in all fusion-positive tumours, whereas all fusion-negative tumours were completely devoid of clear cells. This indicated an association between $M A M L 2$ gene rearrangement and clear cell variants of this tumour [12]. In comparison to this study, investigations by Kyrpychova et al. showed no correlations between translocation-positive hidradenomas and morphological details [19]. In another study, Kuma et al. reported no $M A M L 2$ rearrangement in prominent cystic tumours and poroid hidradenomas [2]. In our study, more or less clear cell component was clearly correlated with FISH status; however, this was not true for all cases (Fig. 2). In one of our cases, a significant clear cell component with FISH-negative status was observed. As mentioned above, fusion-positive tumours were seen in younger patients as compared to negative ones, but the clinical significance remains unclear.

The practical use of MAML2 and EWSR1 rearrangements in skin tumours has not been well established. Requena and Sangüeza revealed the presence 
of $M A M L 2$ translocation in apocrine hidradenomas [24]. This translocation may be helpful in the differential diagnosis of poromatous lesions including poroid (eccrine) hidradenoma. Because EWSR 1 rearrangements have been described in mixed tumours, myoepithelioma and myoepithelial carcinoma, it can be used for the differential diagnosis of these lesions with hidradenomas [4]. In salivary gland tumours, showing $M A M L 2$ and EWSR1 rearrangements is very helpful for distinguishing between MEC and CCC in daily practice $[13,17,20]$.

Despite Moller's article, we did not observe any EWSR 1 FISH positivity in our study group that included $M A M L 2$ negative ones, similarly to many other studies [4]. The results of our study support the theory that $M A M L 2$ is a marker for hidradenomas particularly the clear cell variant. Furthermore, EWSR1 study seems to be obligatory for correct diagnosis of $M A M L 2$-negative MECs, especially those originating from minor salivary glands, to exclude the possibility of a closed mimic CCCs.

The authors would like to thank to Prof. Dr. Ömer Günhan (MD. Head and Neck Pathologist) for his kind belp to analyse the EWSR1 positive cases; technician Yasemin Eskirecepoğlu and molecular biologist Merve Gizem Sezginer for their technical assistance and Enago (www. enago.com) for the English language review.

The authors declare no conflict of interest.

\section{References}

1. Nandeesh BN, Rajalakshmi T. A study of histopathologic spectrum of nodular hidradenoma. Am J Dermatopathol 2012; 34: 461-470.

2. Kuma Y, Yamada Y, Yamamoto $H$, et al. A novel fusion gene CRTC3-MAML2 in hidradenoma: histopathological significance. Hum Pathol 2017; 70: 55-61.

3. Möller E, Stenman G, Mandahl N, et al. POU5F1, encoding a key regulator of stem cell pluripotency, is fused to EWSR1 in hidradenoma of the skin and mucoepidermoid carcinoma of the salivary glands. J Pathol 2008; 215: 78-86.

4. Antonescu CR, Zhang L, Chang NE, et al. EWSR1-POU5F1 fusion in soft tissue myoepithelial tumors. A molecular analysis of sixty-six cases, including soft tissue, bone, and visceral lesions, showing common involvement of the EWSR1 gene. Genes Chromosomes Cancer 2010; 49: 1114-1124.

5. Hsieh MS, Wang H, Lee YH, et al. Reevaluation of MAML2 fusion-negative mucoepidermoid carcinoma: a subgroup being actually hyalinizing clear cell carcinoma of the salivary gland with EWSR1 translocation. Hum Pathol 2017; 61: 9-18.

6. Zhao YN, Wang X, Liang FH, et al. Hyalinizing clear cell carcinoma of salivary glands: A retrospective study focused on uncommon morphology, immunohistochemistry, and detection of gene fusion using fluorescence in situ hybridization. Pathol Res Pract 2018; 214: 380-384.

7. Elder DE, et al. WHO Classification of Skin Tumours. 4th ed. 2018. IARC Press, Lyon 2018.

8. Qannam A, Bello IO. Comparison of histological grading methods in mucoepidermoid carcinoma of minor salivary glands. Indian J Pathol Microbiol 2016; 59: 457-462.
9. Russell-Goldman E, Dubuc A, Hanna J. Differential Expression of PLAG1 in Apocrine and Eccrine Cutaneous Mixed Tumors: Evidence for Distinct Molecular Pathogenesis. Am J Dermatopathol 2020; 42: 251-257.

10. Andersson MK, Kölby L, Nilsson JA, Stenman G. Clinical, genetic and experimental studies of the Brooke-Spiegler (CYLD) skin tumor syndrome. J Plast Surg Hand Surg 2019; 53: 71-75.

11. Amin SM, Beattie A, Ling X, et al. Primary Cutaneous Mammary Analog Secretory Carcinoma With ETV6-NTRK3 Translocation. Am J Dermatopathol 2016; 38: 842-845.

12. Winnes M, Mölne L, Suurküla M, et al. Frequent fusion of the CRTC1 and MAML2 genes in clear cell variants of cutaneous hidradenomas. Genes Chromosomes Cancer 2007; 46: 559-563.

13. Skálová A, Stenman G, Simpson RHW, et al. The Role of Molecular Testing in the Differential Diagnosis of Salivary Gland Carcinomas. Am J Surg Pathol 2018; 42: e11-e27.

14. Noujaim J, Jones RL, Swansbury J, et al. The spectrum of EWSR1-rearranged neoplasms at a tertiary sarcoma centre; assessing 772 tumour specimens and the value of current ancillary molecular diagnostic modalities. Br J Cancer 2017; 116: 669-678.

15. Skálová A, Weinreb I, Hyrcza M, et al. Clear cell myoepithelial carcinoma of salivary glands showing EWSR1 rearrangement: molecular analysis of 94 salivary gland carcinomas with prominent clear cell component. Am J Surg Pathol 2015; 39: 338-348.

16. Tanguay J, Weinreb I. What the EWSR1-ATF1 fusion has taught us about hyalinizing clear cell carcinoma. Head Neck Pathol 2013; 7: 28-34

17. Shah AA, LeGallo RD, van Zante A, et al. EWSR1 genetic rearrangements in salivary gland tumors: a specific and very common feature of hyalinizing clear cell carcinoma. Am J Surg Pathol 2013; 37: 571-578.

18. Flucke U, Palmedo G, Blankenhorn N, et al. EWSR1 gene rearrangement occurs in a subset of cutaneous myoepithelial tumors: a study of 18 cases. Mod Pathol 2011; 24: 1444-1450.

19. Kyrpychova L, Kacerovska D, Vanecek T, et al. Cutaneous hidradenoma: a study of 21 neoplasms revealing neither correlation between the cellular composition and CRTC1-MAML2 fusions nor presence of CRTC3-MAML2 fusions. Ann Diagn Pathol 2016; 23: 8-13.

20. Saade RE, Bell D, Garcia J, et al. Role of CRTC1/MAML2 Translocation in the Prognosis and Clinical Outcomes of $\mathrm{Mu}-$ coepidermoid Carcinoma. JAMA Otolaryngol Head Neck Surg 2016; 142: 234-240.

21. Gorunova L, Mertens F, Mandahl N, et al. Cytogenetic heterogeneity in a clear cell hidradenoma of the skin. Cancer Genet Cytogenet 1994; 77: 26-32.

22. Behboudi A, Winnes M, Gorunova L, et al. Clear cell hidradenoma of the skin - a third tumor type with a $\mathrm{t}(11 ; 19)$ - associated TORC1-MAML2 gene fusion. Genes Chromosomes Cancer 2005; 43: 202-205.

23. Kazakov DV, Ivan D, Kutzner H, et al. Cutaneous hidradenocarcinoma: a clinicopathological, immunohistochemical, and molecular biologic study of 14 cases, including Her2/neu gene expression/amplification, TP53 gene mutation analysis, and $\mathrm{t}(11 ; 19)$ translocation. Am J Dermatopathol 2009; 31: 236-247.

24. Requena L, Sangüeza O. Apocrine Hidradenoma, in Cutaneous Adnexal Neoplasms. Requena L, Sangüeza O (ed.). Springer International Publishing, Cham 2017; 81-103.

\section{Address for correspondence}

\section{Onder Bozdogan}

Department of Pathology, Gulhane Education and Research Hospital Etlik, Ankara, Turkey

e-mail: uplpa04@hotmail.com 